\title{
Distance Education and Social Learning in e-Health
}

\author{
P. Di Bitonto, F. Di Tria, T. Roselli, V. Rossano, and F. Berni
}

\begin{abstract}
In the context of chronic diseases, in order to guarantee a good quality of life, education is as important as the patient's adherence to a specific therapy. Learning and training processes in these contexts has a dual purpose, on the one hand, to make the patient empowered in order to allow him to recognize the behaviors that might compromise her/his health and, secondly, to train non-specialist medical and paramedical staffs on the procedures for diagnosis and patients follow-up to provide an high quality service. In this context the UBICARE project aims at creating a system that favors the de-hospitalization of patients suffering from peritoneal dialysis and chronic heart failure. The paper describes how the e-learning can be fruitful for patient and medical staff training.
\end{abstract}

Index Terms - Distance education, social learning, e-health.

\section{INTRODUCTION}

In the context of chronic diseases to ensure a good quality of life, education and information is as important as adherence to a specific therapy. One of the main objectives of the UBICARE (UBIquitous knowledge-oriented HealthCARE) project is to design and build a system that favors the de-hospitalization of patients suffering from peritoneal dialysis and chronic heart failure. The output of the project will be a social network that will provide a wide range of services such as: sharing clinical data among different care levels (hospital general departments, social and health districts), supporting the medical and paramedical staff in the diagnosis and monitoring of patients, empowering the patients, training of medical and paramedical staff about diagnostic procedures, therapeutic interventions and follow-up of patients.

E-learning is one of the main challenging aspects of the project for training both the patient and the medical staff. Since, in medicine the training and learning process should be personalized, contextualized and quick, in the UBICARE system there is a component that implements the situated learning aiming at selecting and suggesting the most suitable resources for the user based on the user learning needs and the context of use.

The main purpose of this paper is to describe the UBICARE system component and to discuss about the

Manuscript received June 19; revised September 16, 2013. This work was supported in part by the Project UBICARE (UBIquitous knowledge-oriented HealthCARE) - EU-FESR P.O. Puglia Region 2007-2013 Grant in Support of Regional Partnerships for Innovation - Investing in your future (UE-FESR P.O. Regione Puglia 2007-2013 - Asse I - Linea 1.2 - Azione 1.2.4 - Bando Aiuti a Sostegno dei Partenariati Regionali per l'Innovazione - Investiamo nel vostro futuro).

The authors are with Department of Computer Science, University of Bari, Via Orabona, 4, 70125 Bari, Italy (e-mail: dibitonto@di.uniba.it, francesco.ditria@uniba.it,_teresa.roselli@uniba.it, veronica.rossano@uniba.it, flora.berni@uniba.it).
Knowledge Management Service component addressed to manage all the learning resources. In particular, the paper presents the different user profiles and resources involved in the e-learning component of the system.

\section{THE UBICARE SYSTEM}

The UBICARE project aims to design, develop and test a social network framework, which using semantic ontology and knowledge management technologies, support the "Hub and Spoke" health care models. The hub-and-spoke distribution paradigm (or model or network) is a system of connections arranged like a chariot wheel, in which all traffic moves along spokes connected to the hub at the center. The Hub-and-Spoke system is the standard for most major airlines around the world to save airlines money and give passengers better routes to destination.

The model is becoming popular for hospital networks [1], [2], [3], where a single specialized center (Hub) is specialized for the treatment of a specific disease and focused on health care, supported by a network of services (Spokes) to transport the patients to reach the minimum severity levels required to take advantage of the specific treatment. In this context, the UBICARE system aims at offering innovative solutions to support sharing of data, information, and protocols among hub and spoke centers. The ultimate goal is to make the spoke centers able to follow the patient and to manage the low and medium critical situations, sending the patient to the hub center only in the case of serious problems.

In particular, the main services offered by the UBICARE system are:

- A Social Network able to provide various services (training, support to medical activities, patient monitoring) to operators as well as to patients;

- A Decision Support Service able to analyze medical records in real time, recognize the patient, associate the most appropriate treatment protocols, monitor the patients health, send alert if a critical situation occurs;

- A Knowledge Management to oversee e-learning activities and allow the sharing of educational resources among the community users.

Since the system will be a social network that will offer different services and functionalities addressed to distance education and to remote management of patients, a popular open sources framework for social network has been adopted: Elgg [4]. The plug-in architecture of Elgg allows the system to be personalized in order to meet the project goals. In particular, as showed in Fig. 1 the main component of the system are the: Health Care Network (1); Data Acquisition (2); Patient Monitoring (3); Knowledge Management Service (4); Decision Support System (5); Data Service Gateway (6). 


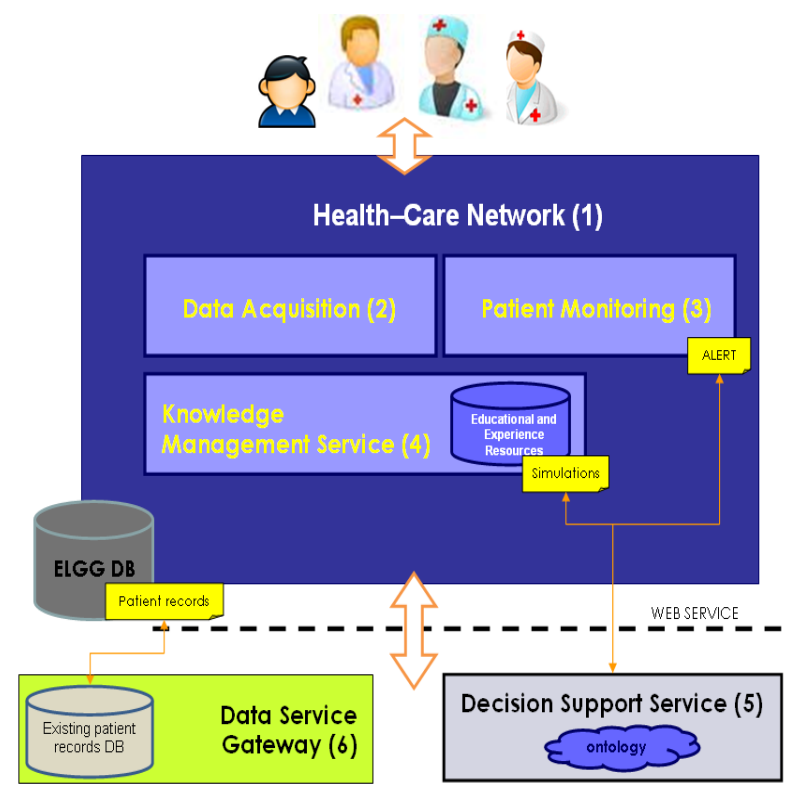

Fig. 1. The architecture of UBICARE system.

\section{A. Health-Care Network (HCN)}

The HCN represents the multimodal interface of the whole system. The plug-in architecture allows a high grade of scalability in terms of both services and functionalities.

\section{B. Data Acquisition}

Data collected by the system will be used for patient's management and monitoring. In order to preserve the accuracy and the continuous updating of data, different data entry modalities have been defined:

- Automatic acquisition using medical devices;

- Manual acquisition of parameters monitored daily by the patient/doctor/paramedic.

\section{Patient Monitoring}

The distance patient monitoring allow doctors and caregivers to be continuously informed about the health conditions of the patient. In particular, the plug-in offers:

- Patient history to summarise the characteristics of the disease and therapy to follow its evolution;

- Automatic alert services to remind regularly controls and to point out critical events;

- Manual alert to signalize critical events for the patient health;

\section{Decision Support Service}

The DSS supports the different users to manage ordinary and extraordinary events. Using data mining algorithms and ontology the DSS is able to suggest alternative therapies; to send automatic alerts based on patient monitoring; to select interesting case studies, on the basis of real cases, to be used in learning and training process.

\section{E. Data Service Gateway}

The Data Service Gateway (DSG) is a service infrastructure that allows the integration and interoperability of data in the whole system. The component ensures the extensibility of the system, the decoupling between distributed components, and the transparency of the communication model and the integration of the system with all different management systems of existing patient records.

\section{THE E-LEARNING COMPONENT}

The KMS is the e-learning component in the UBICARE system, it manages all the learning activities and resources in the system. In particular, the services available are the suggestion of personalised learning resources, serious game to train learners to deal with the main problems of the chronic diseases in the ambit of the project, and the management of experience to share information about both official guidelines and medical protocols.

In detail, the Situated Learning offers personalized learning resources on the basis of the user profile, the context of use and the device. In other words, in order to suggest the best learning activities for the user, a content-based recommender system [5], [6] has been integrated in a search engine. In this way, the best resources for the profile, for the context of use and/or for the device are supplied to the user. Moreover, the recommender system is able to suggest, for each resource chosen by the user, a set of co-related resources that can give more details about the topic.

The Edugames use the simulation approach to train the different user profiles to manage daily activities of the patient, the therapy and the critical events. In particular, the edugames interact with the DSS component that supply real clinical cases that the user is asked to resolve. In accordance with the user's answer a feedback will be proposed. If the user intervention is not correct, some learning resources will be suggested.

The Experiences Manager supports the sharing of best practices among practitioners. The component allows the users to share clinical experiences with her/his colleagues in the social network or to access to resources for professional updates and international official guidelines. S/he can be interested in Q\&A resources in order to discuss with colleagues on a particularly difficult case to find the best solution.

In order to meet these requirements different learner profiles and kind of resources have been defined.

\section{A. Learner Profiles}

The leading expert of the disease is the Hospital practitioner. S/he does not need any kind of training or learning, thus $\mathrm{s} / \mathrm{he}$ is interested in using the Experiences Manager component in order to access to international guidelines for professional updating and discuss about open problems or critical cases.

The Nurses, or professional health workers, who assist the patient during hospitalization and at home. They have to be empowered to take care of patients according to standard protocols and to manage any critical state of health of the patient. Moreover, the nurses should train both patients and families.

The General Practitioner has access to the standard guidelines about diagnostic and therapeutic protocols in order to support patients even in case of unexpected events. In this way, the patient will refer to the specialist only if necessary.

The Patients and their families are trained in the hospital during their stay in order to acquire all the necessary skills about the medical devices and a healthy lifestyle that should be adopted at home. The system, thus, offers learning paths 
that can be useful in order to face potentially critical situations supporting the patients and their families in making the right choice.

\section{B. The Resources for e-Learning}

In order to supply the best learning resources and paths for each user profile, different kind of resources should be managed by the system. In particular, the resources have been classified in resources for Learning and resources to share Experiences.

\section{1) Learning resources}

The Learning Resources are both Learning Objects (LO) and Edugames. Traditional forms of education have been enriched with more interactive forms to motivate and help users in order to maintain higher levels of interest.

The Edugames are simulations that, using the learning-by-doing approach [7], allow specific skills related to both treatment protocols and the possible actions to take in emergency situations to be acquired. In particular, starting from medical records on the basis of the user profile, a problem is automatically defined. The user answer is compared with the DSS solution and if they are different a set of learning resources to fill the learning gap are supplied.
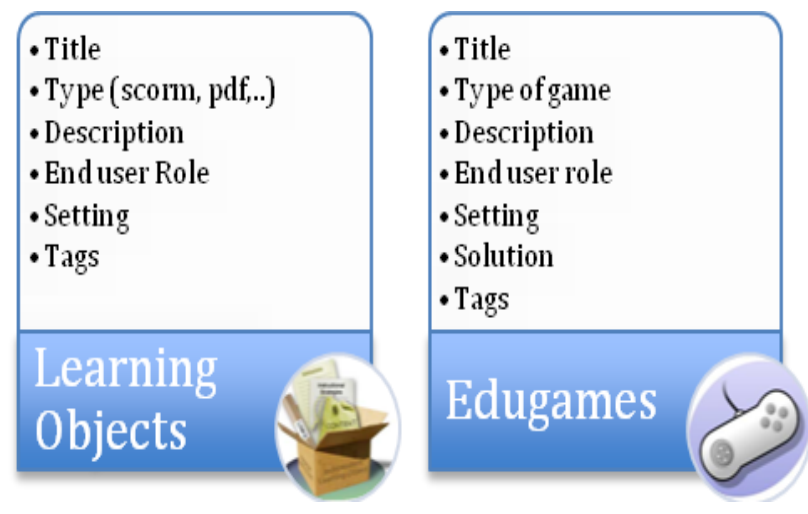

Fig. 2. Learning resources metadata.

In order to build personalised and contextualised learning paths the resources in the system are described using a set of metadata defined for the project, which contains some common and some specific metadata for each kind of resource. The common metadata are the End-User Role, Setting (nephrology or cardiology) and Tags useful to the recommender system to select the resources on the basis of the user profile and the context of use. The specific metadata are useful to filter and order the resources on the basis of the utility for the specific user.

\section{2) Experience resources}

The Experience resources are those resources that the community of practice shares. In the context of the project Links, Best practices and Questions \& Answers (Q\&A) have been considered the most interesting.

The Links are bookmarks for professional updating, Best Practices are both official guidelines for diagnosis and treatment protocols, and hospital practitioner reports that describe clinical cases for which there are no official and recognized references. These resources can be enriched with multimedia files in order to describe the clinical case more precisely. Thus, the Best practices can support the hospital practitioner during the diagnosis or treatment definition for new or difficult clinical cases. The Q\&As allow the community to discuss about open problems. The answers can be evaluated by the community enriching the knowledge in the UBICARE system. The details about metadata for Experience Resources are summarized in Fig. 3.

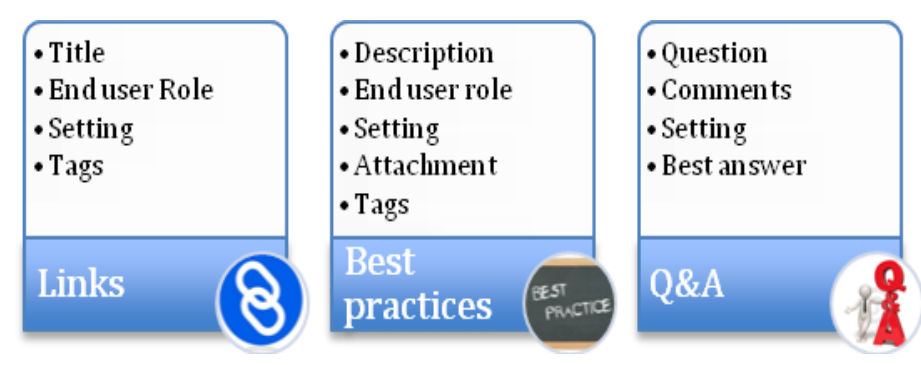

Fig. 3. Experience resources metadata.

In order for learning to be effective, the definition of Learner and resources relationships were defined. In Fig. 4 details are given. The learning resources are addressed mainly to patients, families and nurses. Also, general practitioners can access the learning resources and link in order to be constantly updated. The experience resources are, instead, addressed to only the hospital practitioners.

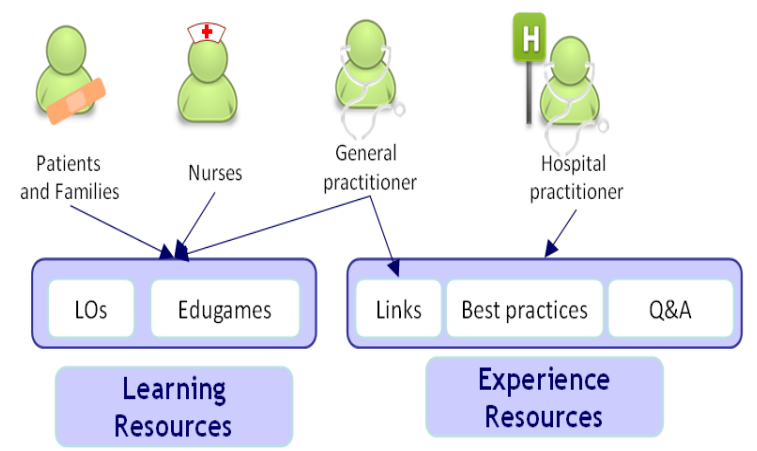

Fig. 4. Learner-resources relationship.

\section{THE USER-SYSTEM INTERACTION}

In order to show the system, the hospital practitioner interaction is described herein. As previously discussed, this profile does not need any kind of learning, but s/he is interested in all resources useful for professional updating. In particular, sharing of experiences is very useful in order to treat difficult cases. For this reason, in the UBICARE system both official documents and best practices are available.

The KMS interface appears as a search engine interface, but the functionalities are the same as a recommender system that is able to suggest resources on the basis of both resource metadata and user features.

The content-based method, in fact, is able to suggest in addition to the resources that meet the user's request, a set of related resources that have a semantic correlation with the one selected by the user.

Fig. 5 shows the page in which the user can start the search. A first recommendation is shown as "Suggested for you" ("Suggeriti per te" in the figure) that lists, using a demographic method, the resources appreciated by similar users to the current one. When a search is done, the 
content-based method selects all the resources that are suitable for the user profile and for her/his learning needs.

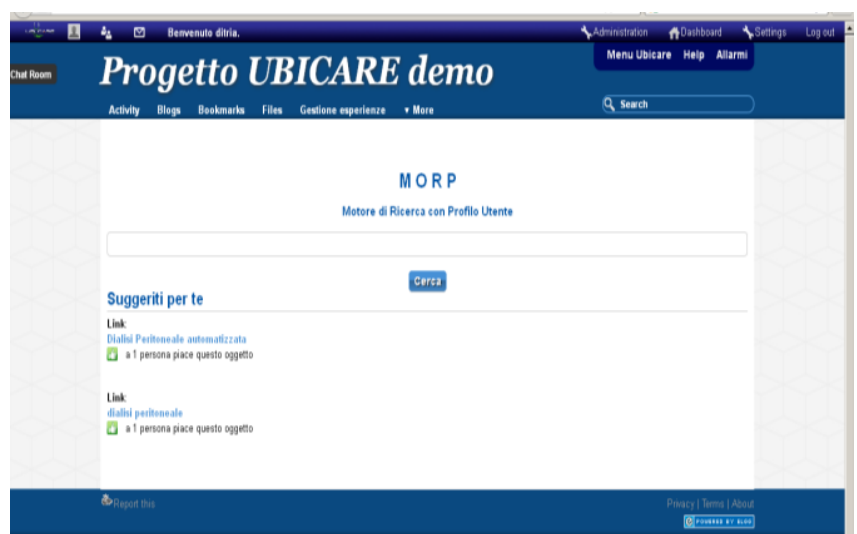

Fig. 5. The search engine page ("Suggeriti per te" is the results of suggestion of the recommender system).

Moreover, for each resource selected by the user, the content-based method selects a set of related resources on the basis of the semantic correlation with the current one ("Oggetti correlati" in Fig. 6).

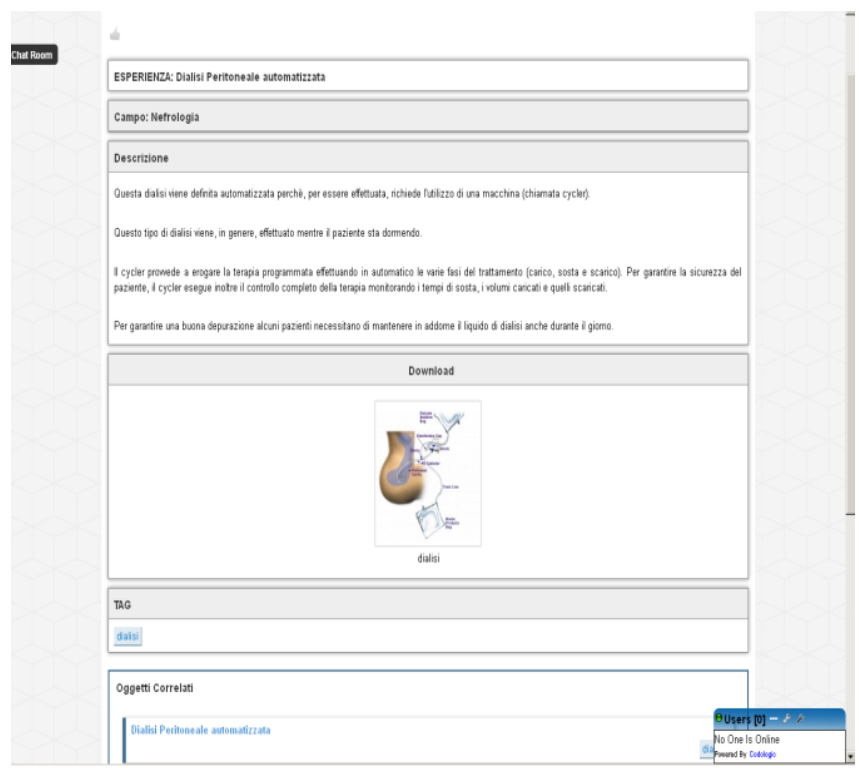

Fig. 6. The co-related resources (in the box "Oggetti Correlati”).

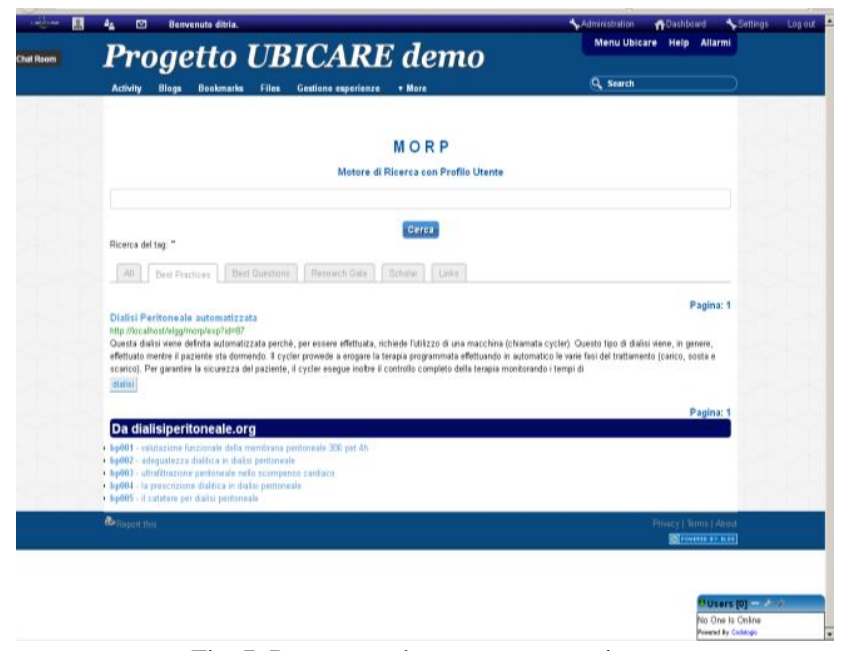

Fig. 7. Recommender system suggestions.

If the user appreciates it, the resources can use the Like button to allow her/his appreciation to be used in next recommendations to predict how much a resource can be suitable for her/his profile and learning needs.

The recommendation list includes all available resources for the user profile. For each type of resource the recommender system makes a suggestion. Using the navigation tab, the user can select the type of resources to view. Moreover, when the user makes a search the KMS sends a request to the most used international search engine for scientific publications such as Google Scholar and Research Gate (Fig. 7). In addition, search results include the best practices retrieved from the Italian official site about peritoneal dialysis (dialisiperitoneale.org).

\section{CONCLUSIONS AND FUTURE WORKS}

It is a common belief that a reduction in health care spending must correspond to a reduction in the quality of care service. The use of new technologies for health care can mitigate this by providing innovative solutions able to maintain high level of quality at a low cost. This is the main challenge of the UBICARE project that aims at supporting the Hub \& Spoke model in medicine. The aim is to support the empowerment of local hospitals, general practitioners, nurses and families, which can take care of the patient even in critical situations. The KMS component allows to manage all the resources that can support the empowerment process. Among the learning services offered there is the sharing of best practices that can support the hospital practitioner in making diagnosis and define a treatment. The recommender system integrated in the KMS allows the best suited resources (Links, Best practices, Q\&As) to the user's profile and the context of use.

In the next future, the recommender system should be refined using another approach in order to enrich the suggestions. Moreover, also information about geo-location will be added, in order to offer resources that are contextualized according to the context in which the request is sent.

\section{REFERENCES}

[1] L. Nobilio and C. Ugolini, "Selective referrals in a "hub and spoke' institutional setting: the case of coronary angioplasty procedures," Health Policy, vol. 63, no. 1, pp. 95-107, January 2003.

[2] J. Stensland, S. M. Speedie, M. Ideker, J. House, and T. Thompson, "The Relative Cost of Outpatient Telemedicine Services," Telemedicine Journal, vol. 5, no. 3, pp. 245-256, September 1999.

[3] W. T. Lee, E. F. Renaud, and O. Hills, "Emergency Psychiatry: An Emergency Treatment Hub-and-Spoke Model for Psychiatric Emergency Services," Psychiatric Services, vol. 54, no. 12, December 2003.

[4] Open source framework. ELGG. [Online]. Available: http://elgg.org/.

[5] B. Robin, "Hybrid Recommender Systems: Survey and Experiments," User Modeling and User-Adapted Interaction, vol. 12, no. 4, pp. 331-370, November 2002.

[6] F. Ricci, L. Rokach, B. Shapira, and P. B. Ed. Kantor, Recommender Systems Handbook, New York: Springer-Verlag, 2011.

[7] R. C. Schank, T. R. Berman, and K. A. Macperson, "Learning by doing," in Instructional design theories and models: A new paradigm of instructional theory, C. M. Reigeluth, Ed., vol. II, Mahwah, NJ: Lawrence Erlbaum Associates, pp. 161-181, 1999, 


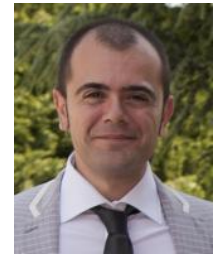

Pierpaolo Di Bitonto has got the bachelor degree in computer science at the University of Bari in October 2002, the Master degree in Computer Science at the University of Bari in March 2004, the $\mathrm{PhD}$ in Computer Science at the University of Bari in May 2009.

He had a Post-Doc fellowship in 2008-2009. His research interests are in, recommender systems and multi-agent system (MAS) particularly in e-learning and tourism. He is co-author of about 40 papers published in journals, conferences and collections (national and international).

Dr. Di Bitonto during the $\mathrm{PhD}$ program he addressed theoretical and methodological issues, also designing, developing and testing software tools. The work has begun with the definition of a multiagent architecture for the student profiling on the basis of the tracking data of the e-learning platform and the learning object personalization. Both the papers presented in the International Workshop on Distance Education Technologies, 2011 and 2012, were awarded of the First place award.

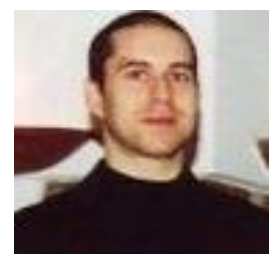

Francesco Di Tria received his Laurea degree in informatics in 2007 from University of Bari "Aldo Moro". In 2011, he received the $\mathrm{PhD}$ in Informatics from the same University. Currently, he is a research fellow at the Computer Science Department. He teaches courses of "Databases" and "Information Systems" for the computer science curriculum. His research interests are in data warehousing and business intelligence systems. In detail, his main activity regards the automation of processes for data warehouse modeling and the definition of metrics for data warehouse quality measurement.

Dr. Di Tia is author and/or co-author of over 20 papers published in international journals, books, and conferences and has participated to different research projects aiming at designing and developing innovative solutions in the field of intangible cultural heritage and e-health.

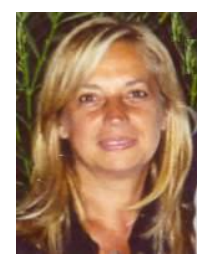

Teresa Roselli has got the degree in computer science (MS) at the University of Bari (Italy) in March 1978. Her research interests are addressed to different e-learning fields both in web and multimedial environments, and in particular to themes such as: the user/student modeling, the interaction of e-learning processes, the sharing of e-learning resources, the multi-agents systems, the semantic web, and recommender systems.

She is an associate professor at the University of Bari "A. Moro" (Italy) since March 2002. She chairs the Multimedial Laboratory, named ADA, of Faculty of Science; she is member of the e-learning Committee of the University of Bari.

Prof. Roselli's efforts have been focused to create relationships among School, University and Business World and, for this purpose, in addition to the activities for the degree courses, she organized in 2001, 2008 and 2012 the Italian Conference on Educational Technology named
"DIDAMATICA". She is author of over one hundred of publications including journal and conference papers. She is part of the editorial board of Journal of e-learning and Knowledge Society (Je-LKS), is referee of the International Journal of Distance Education Technologies (IJDET) e per Journal of Educational Technology \& Society. She is part of international and national program committees, and she was general chair of national and international conferences and workshops. Both the papers presented in the International Workshop on Distance Education Technologies, 2011 and 2012, were awarded of the First place award. She was supervisor of different $\mathrm{PhD}$ thesis on e-learning and recommender systems fields.

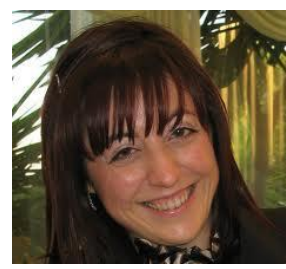

Veronica Rossano has got the degree in computer science (MS) at the University of Bari (Italy) in December 2001 and took the $\mathrm{PhD}$ in Informatics at the University of Bari (Italy) discussing a dissertation titled "From Content Objects to Learning Objects: Adding Instructional and Experience-based Information to Educational Metadata" (supervisor prof. Teresa Roselli) in May 2006.

She is an assistant professor at the University of Bari "A. Moro" starting from May 2006. Her research interests are addressed to the e-learning field, and in particular, to the definition of methodologies, specifications and software that can support educational processes and can allow a high level of didactic resource reuse and an adequate level of personalisation of learning path.

Dr. Rossano is author of many journal and conference papers, both at national and international level, and she is part of international and nationa program committees. She is component of the International Editorial Review Board of: Journal of Distance Education Technologies (IJDET) and Journal of e-learning and Knowledge Society (Je-LKS). Both the papers presented in the International Workshop on Distance Education Technologies, 2011 and 2012, were awarded of the First place award.

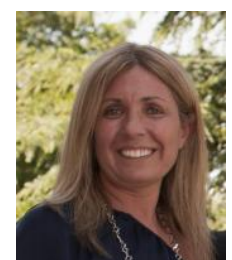

Flora Berni has got the degree in education (MS) at the University of Bari (Italy) in March 2002. She attended an Advanced Professional Training Courses in Designer for Distance Learning in 2004

She was contract professor for the teaching of Italian Language: Communication Techniques in the bachelor degree course on Informatics and Digital Communication; since 2004 she worked in Multimedial Laboratory, named ADA, of Faculty of Science that supports the e-learning activities for Faculty of Science where she is instructional designer and e-learning platforms administrator. Currently she holds a contract for the UBICARE project.

Mrs Berni has been part of the scientific secretariat of DIDAMATICA 2008 and 2012. She is author of some papers in Educational Technology. 